\title{
Limited expression of cancer-testis antigens in renal cell carcinoma patients
}

\author{
NORIHITO SOGA ${ }^{1}$, YASUHIDE HORI ${ }^{2}$, KOICHIRO YAMAKADO $^{3}$, HIROAKI IKEDA ${ }^{4}$, \\ NAOKO IMAI ${ }^{4}$, SHINICHI KAGEYAMA ${ }^{4}$, KAZUNORI NAKASE ${ }^{6}$, ATSUSHI YUTA ${ }^{7}$, \\ NORIO HAYASHI $^{1}$, HIROSHI SHIKU ${ }^{4,5}$ and YOSHIKI SUGIMURA ${ }^{2}$ \\ ${ }^{1}$ Division of Urology, Aichi Cancer Center Hospital, Nagoya, Aichi 464-8681; Divisions of ${ }^{2}$ Nephro-Urologic Surgery \\ and ${ }^{3}$ Radiology, Departments of ${ }^{4}$ Immuno-Gene Therapy, ${ }^{5}$ Cancer Vaccine, ${ }^{6}$ Cancer Center and ${ }^{7}$ Otorhinolaryngology, \\ Head and Neck Surgery, Mie University Graduate School of Medicine, Tsu, Mie 514-8507, Japan
}

Received May 11, 2012; Accepted October 31, 2012

DOI: $10.3892 / \mathrm{mco} .2012 .40$

\begin{abstract}
The aim of this study was to evaluate the frequency of expression of the cancer-testis antigens (CTAs) NY-ESO-1, MAGE-A4 and SAGE, in renal cell carcinoma (RCC) patients compared to that in head and neck cancer (HNC) patients, which represent a positive control with a high incidence of CTA expression, to identify novel target antigens for immunotherapy. We prospectively examined frozen tissue samples collected from surgery or biopsy from 35 RCC and 40 HNC patients. Total RNA was extracted, and real-time reverse transcription-polymerase chain reaction (RT)-PCR was performed to determine the expression of MAGE-A4, NY-ESO-1 and SAGE. MAGE-A4 was not detected in any of the RCC samples, although a low incidence of NY-ESO-1 $(5.7 \% ; 2 / 35)$ and SAGE $(2.9 \% ; 1 / 35)$ expression was observed. No samples demonstrated co-expression of the three CTAs. By contrast, a comparatively high incidence of CTA expression was detected in squamous cell carcinoma (SCC) specimens of HNC patients. The actual incidence was $42.5 \%(17 / 40)$ for MAGE-A4, 20\% (8/40) for NY-ESO-1 and 15\% (6/40) for SAGE. The incidence of co-expression was $7.5 \%(3 / 40)$ for MAGE-A4 and NY-ESO-1, 7.5\% (3/40) for MAGE-A4 and SAGE, 7.5\% (3/40) for NY-ESO-1 and SAGE, and 2.5\% (1/40) for the CTAs. The number of HNC samples positive for MAGE-A4 was significantly higher compared to that of RCC samples. The remaining two antigens, NY-ESO-1 and SAGE, were expressed at high levels in HNC compared to RCC samples. Limited frequency of CTA (NY-ESO-1, MAGE-A4 and SAGE) expression was demonstrated in RCC compared to HNC samples.
\end{abstract}

Correspondence to: Dr Norihito Soga, Division of Urology, Aichi Cancer Center Hospital, 1-1 Kanokodenn, Chigusa-ku, Nagoya, Aichi 464-8681, Japan

E-mail: n-soga@aichi-cc.jp

Key words: cancer-testis antigen, kidney cancer

\section{Introduction}

Renal cell carcinoma (RCC) accounts for $2 \%$ of all types of cancer, and the reported incidence is on the increase due to advances in imaging technology (1). Distant metastases have been reported to develop in $24-30 \%$ of the patients who undergo radical nephrectomy $(2,3)$. RCC patients with metastases who are not surgical candidates receive immunotherapy, chemotherapy or molecular target-based therapy as palliative treatment. However, the survival of these patients is limited. The median survival times reported are 7-8.5 months following immunotherapy, 6-10 months following systemic chemotherapy (4) and $<19.3$ months following molecular target-based therapy $(5,6)$. Therefore, additional therapeutic options for RCC patients with unresectable metastases are needed.

Several novel regimens that use targeted immunotherapeutic strategies, such as cancer vaccines against MN/CA9 (7) or HIFPH3 (8), have been evaluated in RCC cases. Further investigation of these strategies is required for the precise evaluation of their therapeutic efficacy. It is crucial to define appropriate target antigens that are frequently and specifically expressed in RCC to establish effective RCC immunotherapy.

Cancer-testis antigens (CTAs) are particularly attractive targets for immunotherapy, due to their unique expression profiles. While these antigens are highly expressed in adult male germ cells or placenta, they are completely absent from other normal adult tissues and demonstrate aberrant expression in a variety of malignant neoplasms $(9,10)$. As adult male germ cells do not express major histocompatibility complex (MHC) class I, $\mathrm{CD}^{+}$effector cells theoretically ignore these cells (11). MAGE, NY-ESO-1 and SAGE genes exhibit a similar expression pattern, and their immunogenicity as targets for cancer immunotherapy has been well-studied (12-16). In a recent study, we assessed the efficacy of immunotherapy for several types of cancer-targeting CTAs $(17,18)$. As a first step towards the development of effective immunotherapy for RCC patients, it is crucial to evaluate the expression level of CTA in the targeted cancer. However, a limited number of studies has estimated the incidence of CTA expression in RCC cases, with controversial results (19-22). The aim of this study was to precisely evaluate the frequency of CTA expression in RCC with specific and quantitative methodology. 
We performed quantitative real-time reverse transcription-polymerase chain reaction (RT-PCR) for MAGE-A4, NY-ESO-1 and SAGE with specific primers in RCC compared to head and neck cancer (HNC) patients, which represent a positive control with a high incidence of CTA expression, to assess the future potential of CTA-targeted immunotherapy.

\section{Materials and methods}

Cases. Decisions regarding sample analysis were made following discussion with patients and obtaining their informed consent. Informed consent was obtained in accordance with the requirements of our Institutional Review Board (IRB) at the Mie University School of Medicine (Tsu, Japan).

Between September, 2009 and June, 2011, 35 RCC samples were obtained by surgery or biopsy. The 35 patients ( 29 males and 6 females) had a median age of 61.9 years (range, 41-87). The average tumor size was $5.0 \mathrm{~cm}$ (range, 1.8-12.0). Thirty-one patients had undergone radical nephrectomy, while one patient had initially undergone partial nephrectomy. In the remaining 3 cases computed tomography (CT)-guided biopsy was performed to obtain a tumor specimen prior to radiofrequency ablation.

Histological examination demonstrated that the 35 cases were clear cell RCC. Of these, 29 cases were grade $\leq 2,4$ were grade $\geq 3$ and in 2 cases the grade classification was unknown (Table I).

Samples from $40 \mathrm{HNC}$ cases were collected by surgery or biopsy for the positive control with a high incidence of CTA expression between December, 2008 and August, 2011. The 40 patients ( 28 males and 12 females) had a median age of 63.9 years (range, 34-87). The average tumor size was $5.9 \mathrm{~cm}$ (range, 1.3-29.0). Regarding cancer location, there were 23 cases of oral cancer (14 cases of tongue, 5 of buccal mucosal and 4 of gingival cancers), and 10 cases of pharyngeal cancer. There were also 5 cases of laryngeal and 2 of maxillary cancer (Table II). In this group, 31 patients had undergone radical resection, while punch core biopsy was performed in 9 patients to obtain a tumor specimen.

RNA extraction and quantitative RT-PCR. Total RNA was extracted from frozen RCC and HNC tissue samples. Complementary DNA (cDNA) was prepared from $1 \mu \mathrm{g}$ of total RNA using the QuantiTect ${ }^{\circledR}$ Reverse Transcription kit (Qiagen, Hilden, Germany). The quantitative PCR analysis for the expression of MAGE-A4, NY-ESO-1 and SAGE was performed in buffer composed of 1X QuantiTect Multiplex PCR NoROX Master mix (Qiagen), 1X Pre-Developed TaqMan $^{\circledR}$ Assay Reagents Control kits (Applied Biosystems, Carlsbad, CA, USA), and $0.4 \mathrm{mM}$ of cognate primers and $0.2 \mathrm{mM}$ of cognate probe. Following enzyme activation for $15 \mathrm{~min}$ at $95^{\circ} \mathrm{C}, 50$ two-step cycles were performed $(1 \mathrm{~min}$ at $94^{\circ} \mathrm{C}$ and $1 \mathrm{~min}$ at $60^{\circ} \mathrm{C}$ ), using the Mx3000P system (Agilent Technologies, Santa Clara, CA, USA). Sequences of the primers and probes used in the present study were: MAGE-A4 F: 5'-GCAGTAATCCTGCGCGCTAT-3' and R: 5'-CATTGA CCCTGACCACATGCT-3'; probe: 5'-FAM-CTCTGGCT GAAACCA-MGB-3'. NY-ESO-1 F: 5'-GGCTGAATGGA TGCTGCAGA-3' and R: 5'-CTGGAGACAGGAGCTGAT GGA-3; probe: 5'-FAM-TGTGTCCGGCAACATACTGAC
Table I. Baseline characteristics of RCC patients.

\begin{tabular}{|c|c|}
\hline Patient demography & Value \\
\hline Total no. of patients & 35 \\
\hline Age (years), median (range) & $61.9(41-87)$ \\
\hline$<60$ & 14 \\
\hline$\geq 60$ & 21 \\
\hline \multicolumn{2}{|l|}{ Gender } \\
\hline Male & 29 \\
\hline Female & 6 \\
\hline \multicolumn{2}{|l|}{ Laterality } \\
\hline Right & 14 \\
\hline Left & 21 \\
\hline \multicolumn{2}{|l|}{ Mean maximum diameter $(\mathrm{cm})$} \\
\hline Median (range) & $5.0(1.8-12.0)$ \\
\hline \multicolumn{2}{|c|}{ Treatment procedure to obtain specimens } \\
\hline Radical nephrectomy & 31 \\
\hline Partial nephrectomy & 1 \\
\hline Biopsy before RFA & 3 \\
\hline \multicolumn{2}{|l|}{ Histological cell type } \\
\hline Clear cell & 35 \\
\hline \multicolumn{2}{|l|}{ Tumor grade } \\
\hline$\leq 2$ & 29 \\
\hline$\geq 3$ & 4 \\
\hline Unknown & 2 \\
\hline
\end{tabular}

RCC, renal cell cancer; RFA, radiofrequency ablation.

TATCCGA-TAMRA-3'. SAGE F: 5'-TGTCATTCACGATAT CCAGGAG G-3' and R: 5'-GGTGGCATACAATGTCCTG TCAT-3'; probe: 5'-FAM-TGTGTCCGGCAACATACTGA CTATCCGA-TAMRA-3'. The GAPDH copy number was measured using Pre-Developed TaqMan Assay Reagents Control kits (Applied Biosystems). To transform the cycle threshold $(\mathrm{Ct})$ values into absolute mRNA copy numbers, we used a dilution series of linearized plasmid containing each gene insert and constructed a calibration curve. Gene expression was evaluated as positive when the value exceeded 12.2 copies/GAPDH $10^{4}$ copies for MAGE-A4, 5.96 copies/ GAPDH $10^{4}$ copies for NY-ESO-1 and 2.81 copies/GAPDH $10^{4}$ copies for SAGE. These cut-off values were determined as the mean \pm 2 standard deviation (SD) of the expression levels in the cognate normal samples.

Statistical analysis. Differences between groups were assessed with the Chi-square test or direct Fisher's exact test. $\mathrm{P}<0.05$ was considered to indicate a statistically significant difference. Statistical analyses were performed with the SPSS software version 20 (SPSS Japan, Tokyo, Japan).

\section{Results}

CTA expression levels were evaluated using real-time RT-PCR in a total of $35 \mathrm{RCC}$ and $40 \mathrm{HNC}$ cases (a positive control 
Table II. Baseline characteristics of HNC patients.

\begin{tabular}{lc}
\hline Patient demography & Value \\
\hline Total no. of patients & 40 \\
Age (years), median (range) & $63.9(34-87)$ \\
$<60$ & 11 \\
$\geq 60$ & 29 \\
Gender & \\
Male & 28 \\
Female & 12 \\
Mean maximum diameter (cm) & \\
Median (range) & $5.9(1.3-29.0)$ \\
Location of tumor & \\
Oral cancers & 23 \\
Tongues & 14 \\
Buccal mucosal & 5 \\
Gingival & 4 \\
Pharyngeal cancer & 10 \\
Laryngeal cancer & 5 \\
Maxillary cancer & 2 \\
Procedure to obtain specimens & \\
Resection & 31 \\
Biopsy & 9 \\
Histological cell type & \\
Squamous cell carcinoma & \\
\hline
\end{tabular}

HNC, head and neck cancer.

with a high incidence of CTA expression). MAGE-A4 was not detected in any of the RCC cases, although a low incidence of NY-ESO-1 $(5.7 \% ; 2 / 35)$ and SAGE $(2.9 \% ; 1 / 35)$ expression was observed. No case demonstrated co-expression of the three CTAs investigated.

By contrast, a comparatively high incidence of expression was detected in squamous cell carcinoma (SCC) specimens of HNC patients. The actual incidence was $42.5 \%$ (17/40) for MAGE-A4, 20\% (8/40) for NY-ESO-1 and 15\% (6/40) for SAGE. The incidence of co-expression was $7.5 \%(3 / 40)$ for MAGE-A4 and NY-ESO-1, 7.5\% (3/40) for MAGE-A4 and SAGE, $7.5 \%$ (3/40) for NY-ESO-1 and SAGE, as well as $2.5 \%$ $(1 / 40)$ for the CTAs.

The number of HNC samples that were positive for MAGE-A4 was significantly higher compared to that of RCC samples $(\mathrm{P}<0.001)$. The remaining two antigens, NY-ESO-1 and SAGE, demonstrated higher expression levels in NSC compared to RCC samples (Table III).

\section{Discussion}

Since CTAs are expressed in tumors and not in normal tissue (except for testicular tissue), CTAs are optimal antigens for cancer-specific vaccines (23). As the first step in the strategy of designing a cancer vaccine, knowledge of the frequency of expression of each CTA in the targeted cancer is crucial.
Table III. Frequency of CTA expression in each type of cancer.

\begin{tabular}{lccc}
\hline Antigen & $\begin{array}{c}\mathrm{RCC}(\mathrm{n}=35) \\
\mathrm{n}(\%)\end{array}$ & $\begin{array}{c}\mathrm{HNC}(\mathrm{n}=40) \\
\mathrm{n}(\%)\end{array}$ & P-value \\
\hline $\begin{array}{l}\text { MAGE-A4 } \\
\text { Positive }\end{array}$ & $0(0)$ & $17(42.5)$ & $<0.001$ \\
Negative & $35(100)$ & $23(57.5)$ & \\
NY-ESO-1 & & & \\
Positive & $2(5.7)$ & $8(20)$ & 0.09 \\
Negative & $33(94.3)$ & $32(80.0)$ & \\
SAGE & & & \\
Positive & $1(2.9)$ & $6(15.0)$ & 0.11 \\
Negative & $34(97.1)$ & $34(85.0)$ & \\
\hline
\end{tabular}

CTAs, cancer-testis antigens. RCC, renal cell carcinoma; HNC, head and neck cancer.

CTA expression is detected using several methods, including immunohistochemistry, RT-PCR and real-time RT-PCR. Among these methods, real-time RT-PCR is able to quantify the RNA expression of the targeted protein, contributing to a highly sensitive outcome.

In the present study, the antigens MAGA-A4, NY-ESO-1 and SAGE demonstrated a low incidence of expression in RCC, but a comparably high incidence of expression in HNC, when assessed with the real-time RT-PCR method.

In previous studies on CTA expression in RCC cases, MAGE-A4 (previously denoted as MAGE4), a member of the MAGEA gene family, was expressed in $30 \%$ (15/50) of the RCC cases (19), while MAGE1 or 2 demonstrated a comparably low incidence of expression based on RT-PCR assay $(19,20)$. However, additional studies showed no expression of MAGE-A4 in 8 RCC cells using RT-PCR (22). MAGE-A4 expression was detected in these cells when they were treated with 5-Aza-CdR. Immunohistochemistry using the monoclonal antibody 57B that reacts with MAGE-A4 and other members of the MAGE family, including MAGE-A1, 3, 6 and 12 , was reported with $0 \%(0 / 9)$ staining with RCC tissues (21). In this study, none of the $35 \mathrm{RCC}$ cases was found to express MAGE-A4 following utilization of specific and quantitative assessment with real-time RT-PCR.

In previous studies, NY-ESO-1 mRNA expression was not detected by RT-PCR in 37 (20), 6 (24) and 10 (12) RCC cases. Our results also suggested a significantly low incidence of NY-ESO-1 expression $(5.7 \%, 2 / 35)$.

To the best of our knowledge, no previous study has evaluated the expression of SAGE in RCC. Our results showed a low incidence of SAGE expression in RCC samples.

Concerning HNC, the reported incidence of MAGE-A4 expression was $60.2-70 \%$ using immunofluorescence $(25,26)$ and $60 \%$ using RT-PCR (27). A similar incidence of $42.5 \%$ (17/40) was found in this study.

NY-ESO-1 was expressed in 9.5-33\% of HNC samples using immunofluorescence $(25,26)$ and $6-6.7 \%$ using RT-PCR $(26,28)$. Our results indicated that $20 \%$ of the $40 \mathrm{HNC}$ cases expressed NY-ESO-1. SAGE was expressed in $27 \%$ of the 
cases using RT-PCR (28), and in $15 \%$ of the HNC cases using real-time RT-PCR

A discrepancy related to CTA expression existed, depending on the method of evaluation (23). In general, immunohistochemical analysis suggested relatively high CTA expression levels compared to RT-PCR (27). This discrepancy may have occurred due to uncoupling of transcription with RT-PCR on the different tissue sections (23). By contrast, the possibility of non-specific binding in immunohistochemical studies cannot be excluded.

To select the tumor types that are optimal candidates for a cancer vaccine based on actual CTA expression, real-time RT-PCR in several sections of each specimen and several evaluation methods, including immunohistochemistry, should be used on the same samples. The immunological response to a cancer vaccine should also be evaluated, depending on the results of CTA expression obtained following the use of various methods.

The meaning of the CTA expression in these tumors, aside from its use in the strategy of designing a cancer vaccine, needs to be further investigated. The correlation between CTA expression and survival or staging has been previously evaluated. Recent studies have indicated that solitary CTA expression was associated with a higher risk of poor outcomes in HNC patients (27). Findings of another study demonstrated NY-ESO-1-positive cases to have a good prognosis, as opposed to MAGE-A-positive cases (26). According to these studies, the correlation between CTA expression and prognosis is controversial, since it depends on the specific CTA assessed. The co-expression of CTA is believed to be based on the order of tumorigenesis (23) or the amount of demethylation, related to the cancer type (29). A high incidence of simultaneous expression of CTA was observed in HNC, and simultaneous expression of CTA tended to correlate with advanced tumor stage, but not survival (25). However, it was difficult to determine a correlation between solitary CTA expression or co-expression with stage or survival, due to the low incidence of CTA expression itself in RCC cases. In the future, the correlation of solitary expression or co-expression of CTA associated with prognosis should be evaluated for various CTAs.

CTA expression was also evaluated as a diagnostic tool, focusing on RCC and differential diagnosis between chromophobe RCC and oncocytoma. The incidence of MAGE-A3/4 expression was $88.2 \%(15 / 17)$ for oncocytoma and $38.9 \%$ (7/18) for chromophobe RCC. In addition, the incidence of NY-ESO-1 expression was $88.2 \%$ (15/17) for oncocytoma and $33.3 \%$ (6/18) for chromophobe RCC (30). In this study, although a small number of cases was evaluated, NY-ESO-1 expression was demonstrated in $33.3 \%$ (1/3) of the chromophobe cases (data not shown), a fact which was in agreement with previous studies (30). We evaluated CTA expression in other types of $\mathrm{RCC}$ to determine its value for predicting poor prognosis or malignancy, including distinguishing between chromophobe RCC and oncocytoma.

Since quantitative real-time RT-PCR provided stable results compared to the methods used previously, our screening method should be useful as the starting point of designing immunotherapy strategies. The results of this study strongly suggest the need to explore additional antigens that are expressed in the same frequency and tumor-specific manner in $\mathrm{RCC}$, for the development of effective immunotherapy of this type of malignancy.

In conclusion, the results of this study have shown that with quantitative real-time PCR, NY-ESO-1, MAGE-A4 and SAGE are expressed at a significantly low frequency in RCC patients. Screening to detect additional CTAs should be continued in the future to develop targeted immunotherapeutic strategies for cancer vaccines in RCC.

\section{Acknowledgements}

Research funding was provided to Hiroaki Ikeda, Naoko Imai and Hiroshi Shiku from Takara Bio, Inc. (Tokyo, Japan). This study was supported by a Grant-in-Aid for Scientific Research and Project for Development of Innovative Research on Cancer Therapeutics from the Ministry of Education, Culture, Sports, Science and Technology of Japan.

\section{References}

1. Hofmann HS, Neef H, Krohe K, Andreev P and Silber RE: Prognostic factors and survival after pulmonary resection of metastatic renal cell carcinoma. Eur Urol 48: 77-82, 2005.

2. Levy DA, Slaton JW, Swanson DA and Dinney CP: Stage specific guidelines for surveillance after radical nephrectomy for local renal cell carcinoma. J Urol 159: 1163-1167, 1998.

3. Ljungberg B, Alamdari FI, Rasmuson T and Roos G: Follow-up guidelines for nonmetastatic renal cell carcinoma based on the occurrence of metastases after radical nephrectomy. BJU Int 84: 405-411, 1999.

4. Pyrhönen S, Salminen E, Ruutu M, et al: Prospective randomized trial of interferon alfa-2a plus vinblastine versus vinblastine alone in patients with advanced renal cell cancer. J Clin Oncol 17: 2859-2867, 1999.

5. Motzer RJ, Michaelson MD, Redman BG, et al: Activity of SU11248, a multitargeted inhibitor of vascular endothelial growth factor receptor and platelet-derived growth factor receptor, in patients with metastatic renal cell carcinoma. J Clin Oncol 24: 16-24, 2006.

6. Escudier B, Eisen T, Stadler WM, et al: Sorafenib in advanced clear-cell renal-cell carcinoma. N Engl J Med 356: 125-134, 2007.

7. Shimizu K, Uemura H, Yoshikawa M, et al: Induction of antigen-specific cellular immunity by vaccination with peptides from MN/CA IX in renal cell carcinoma. Oncol Rep 10: 1307-1311, 2003.

8. Sato E, Torigoe T, Hirohashi Y, et al: Identification of an immunogenic CTL epitope of HIFPH3 for immunotherapy of renal cell carcinoma. Clin Cancer Res 14: 6916-6923, 2008.

9. Boon T and Old LJ: Cancer tumor antigens. Curr Opin Immunol 9: 681-683, 1997.

10. Scanlan MJ, Gure AO, Jungbluth AA, Old LJ and Chen YT: Cancer/testis antigens: an expanding family of targets for cancer immunotherapy. Immunol Rev 188: 22-32, 2002.

11. Uyttenhove C, Godfraind C, Lethé B, et al: The expression of mouse gene P1A in testis does not prevent safe induction of cytolytic T cells against a P1A-encoded tumor antigen. Int J Cancer 70: 349-356, 1997.

12. Jungbluth AA, Chen YT, Stockert E, et al: Immunohistochemical analysis of NY-ESO-1 antigen expression in normal and malignant human tissues. Int J Cancer 92: 856-860, 2001.

13. De Plaen E, Arden K, Traversari C, et al: Structure, chromosomal localization, and expression of 12 genes of the MAGE family. Immunogenetics 40: 360-369, 1994.

14. Chomez P, De Backer O, Bertrand M, De Plaen E, Boon T and Lucas S: An overview of the MAGE gene family with the identification of all human members of the family. Cancer Res 61: 5544-5551, 2001.

15. Chen YT, Scanlan MJ, Sahin U, et al: A testicular antigen aberrantly expressed in human cancers detected by autologous antibody screening. Proc Natl Acad Sci USA 94: 1914-1918, 1997. 
16. Chen YT, Chiu R, Lee P, et al: Chromosome X-encoded cancer/ testis antigens show distinctive expression patterns in developing gonads and in testicular seminoma. Hum Reprod 26: 3232-3243, 2011.

17. Aoki M, Ueda S, Nishikawa $\mathrm{H}$, et al: Antibody responses against NY-ESO-1 and HER2 antigens in patients vaccinated with combinations of cholesteryl pullulan (CHP)-NY-ESO-1 and CHP-HER2 with OK-432. Vaccine 27: 6854-6861, 2009.

18. Shirakura Y, Mizuno Y, Wang L, et al: T-cell receptor gene therapy targeting melanoma-associated antigen-A4 inhibits human tumor growth in non-obese diabetic/SCID $/ \gamma$ cnull mice. Cancer Sci 103: 17-25, 2012.

19. Yamanaka K, Miyake H, Hara I, Gohji K, Arakawa S and Kamidono S: Expression of MAGE genes in renal cell carcinoma. Int J Mol Med 2: 57-60, 1998.

20. Neumann E, Engelsberg A, Decker J, et al: Heterogeneous expression of the tumor-associated antigens RAGE-1, PRAME, and glycoprotein 75 in human renal cell carcinoma: candidates for T-cell-based immunotherapies? Cancer Res 58: 4090-4095, 1998.

21. Jungbluth AA, Busam KJ, Kolb D, et al: Expression of MAGE-antigens in normal tissues and cancer. Int J Cancer 85: 460-465, 2000.

22. Coral S, Sigalotti L, Altomonte M, et al: 5-aza-2'-deoxycytidine-induced expression of functional cancer testis antigen in human renal cell carcinoma: immunotherapeutic implications. Clin Cancer Res 8: 2690-2695, 2002.

23. Kienstra MA, Neel HB, Strome SE and Roche P: Identification of NY-ESO-1, MAGE-1, and MAGE-3 in head and neck squamous cell carcinoma. Head Neck 25: 457-463, 2003.
24. Scanlan MJ, Altorki NK, Gure AO, et al: Expression of cancer-testis antigens in lung cancer: definition of bromodomain testis-specific gene (BRDT) as a new CT gene, CT9. Cancer Lett 150: 155-164, 2000.

25. Figueiredo DL, Mamede RC, Spagnoli GC, et al: High expression of cancer testis antigens MAGE-A, MAGE-C1/CT7, MAGE-C2/CT10, NY-ESO-1, and gage in advanced squamous cell carcinoma of the larynx. Head Neck 33: 702-707, 2010

26. Pastorcic-Grgic M, Sarcevic B, Dosen D, Juretic A, Spagnoli GC and Grgic M: Prognostic value of MAGE-A and NY-ESO-1 expression in pharyngeal cancer. Head Neck 32: 1178-1184, 2010.

27. Cuffel C, Rivals JP, Zaugg Y, et al: Pattern and clinical significance of cancer-testis gene expression in head and neck squamous cell carcinoma. Int J Cancer 128: 2625-2634, 2010.

28. Atanackovic D, Blum I, Cao Y, et al: Expression of cancer-testis antigens as possible targets for antigen-specific immunotherapy in head and neck squamous cell carcinoma. Cancer Biol Ther 5: 1218-1225, 2006.

29. De Smet C, Lurquin C, Lethe B, Martelange V and Boon T: DNA methylation is the primary silencing mechanism for a set of germ line- and tumor-specific genes with a CpG-rich promoter. Mol Cell Biol 19: 7327-7335, 1999.

30. Demirovic A, Dzombeta T, Tomas D, et al: Immunohistochemical expression of tumor antigens MAGE-A3/4 and NY-ESO-1 in renal oncocytoma and chromophobe renal cell carcinoma. Pathol Res Pract 206: 695-699, 2010. 